\title{
Determination of the kinematic parameters of the new constructive solution of the beamless sucker-rod pump
}

\author{
B.B. Ahmedov, A.M. Najafov*, A.I. Abdullayev \\ Azerbaijan Technical University, Department for Machine Design, H. Cavid Ave. 25, AZ 1073 Baku, \\ Azerbaijan
}

\begin{abstract}
The petrol industry has very big impact on the modern world economy and politics. With any options and scenarios for the development of the economy in the coming years, natural resources, especially fossil oil products, will be the main factor of world economic growth. The sucker-rod pumps are one of the important aggregates for the mechanized oil recovery on land. However, the low efficiency, the big expenditure of the electric power, an incomplete construction of driving gear and reformative mechanisms, impossibility of use in existing aggregates of electric motors with a rotation high frequency lead to research of more perfect construction of a mechanical drive pumps. The present study describes a new design solution for a beamless sucker-rod pump for oil production, characterized by small dimensions and metal consumption, less energy consumption, better dynamic characteristics. The results of analytical kinematical studies and numerical calculations of the new beamless sucker-rod pump are presented.
\end{abstract}

\section{Keywords}

Beamless sucker-rod pump; Kinematics; Analytical solution; Duration

Received: 07 September 2018; Accepted: 19 September 2018

ISSN: 2630-5763 (online) (C) 2018 Golden Light Publishing® All rights reserved.

\section{Introduction}

At present, various pump installations are used for the extraction of oil by a mechanized method. In the open literature there are some studies about the history of pumps, production technology and kinematics. Laine et al. [1] presented information on harmonic polished bar motion and production technology of pumping unit. Byrd et al. [2] discussed the issue of history, background and reasons of the oilfield pumping unit. Beck and Peterson [3] compared the performance of linear actuator according to walking pumping systems. Takacs [4] presented full kinematics and torsion analysis of rotaflex pumping units. The main disadvantages of pumps are the large metal capacity of the structure, low efficiency, poor balance, the need for a massive foundation, etc [5]. Of the existing mechanized methods of oil production, the most common is the sucker-rod pump with the balanced individual drives of mechanical action.

Beamless sucker-rod pumps have smaller metal capacity and dimensions compared to balancing. On the other hand, compared to a balancing suckerrod pumps, the beamless has a slightly different movement characteristic of the rod suspension point and a different picture of the forces acting. One of essential deficiencies of known mechanical drives of sucker-rod pumps (beam pumping unit) is

\footnotetext{
Corresponding author

E-mail: ali.najafov@rohe.az
} 
that at the big traverse stroke, except increase in a dimension of the machine tool; raise as well the sizes of a beam head. At the usual beam with application of an arc beam head the span arrow in any position of the mechanism is organized.

Increasing the efficiency of sucker-rod pumps is carried out with the use of more advanced designs and mechanisms. Since the complex approach to the process of improving the sucker-rod pumps is somewhat complicated, it is usually enhanced by the flexibility of the transforming mechanism as well as from its energy saving point of view. One of the important issues when designing the kinematic scheme of the transforming mechanism is to ensure that the suspension point of the shaft is closer to the harmonic law [5].

Operation of the deep pump at each movement plunger up and downwards is accompanied by change of a direction of movement of pump rods and transition through some positions (dead points) with zero speed that should vary during one cycle of operation of machine on magnitude and a direction. At that dynamic loads on rods depend on magnitude of speed and acceleration which are drastic shown at descent of pumps to the big depths [6].

As rods are set in motion by sucker-rod pump, operation of the deep pump essentially depends on the law of movement of a suspension bracket polished rod. Thus, an important task at the oilfields today is to find ways to increase the efficiency and reliability of the sucker-rod pumps in order to reduce operating costs and increase the inter-repair period of well operation. Therefore, the development of a new design solution of beamless mechanical drives for sucker-rod pumps, which eliminates the above drawbacks, is of great practical importance for modern oil engineering. In this study, the solution of the problem is discussed.

\section{Formulation of the problem}

\subsection{A new design of a beamless sucker-rod pump}

The proposed design of the oil pumping unit has an original design and provides the following advantages over other types of oil production equipment:

1. It consumes about 1.5-1.7 times less electricity than conventional sucker-rod pumps.

2. Increases the service life of the gearbox, due to the absence of negative torque on the output shaft.

3. Does not require a solid and high foundation.

4. Less sensitive to the uneven draft of the foundation.

5. There is no massive beam and a bulky rotary head.

6. Due to the work on the symmetrical cycle, the service life of the rod string increases, since dynamic loads and vibrations are greatly reduced.

7. Has small dimensions.

8. It is possible to free space around the wellhead during installation, repair and adjustment of the trajectory of the rod suspension point along a vertical line.

9. It is possible to fully unfold the front and rear racks during the transportation of the rocking machine.

Fig. 1 shows a diagram of a new solution of the beamless sucker-rod pump.

The new solution of the beamless sucker-rod pump contains two cranks 7 , rigidly fixed on both sides at the output ends of the driven shaft of the multi-stage AN-reducer 4, which has only two shafts and gear ratio 1: 125 [7]. At one end of the drive shaft of the AN-reducer, a stepped driven pulley 6 of the V-belt drive 3 is installed, and at the other output end- a two-disk brake 5 is located. The mechanical drive comprises a frame 1 made of profile rolling in the form of two longitudinal crossconnects, two brackets for connecting the front pillars 18, two brackets for connecting the rear pillars 19, and two brackets for connecting the front rods 16 and two brackets for connecting rear rods 17. On the frame together with an AN-reducer is installed a three-phase asynchronous electric motor 2. 


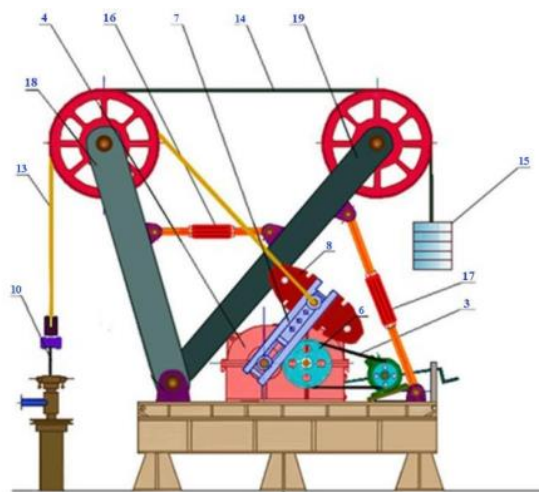

a)

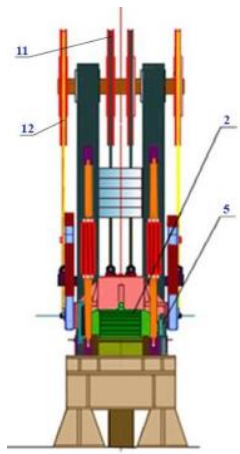

b)

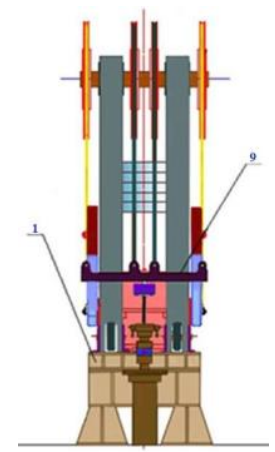

c)

Fig. 1. A diagram of a new solution of the beamless sucker-rod pump

As part of the transforming mechanism of mechanical drive, there are also ropes 13,14 and blocks 11, 12. At one end of the parallel ropes, a crosspiece 9 is suspended from which the column of the rods 10 is fixed and at the other end - cranks with a counterweight 8 are fixed. On the other hand, with the parallel ropes 14, the traverse is connected to a movable counterweight 15 giving a gain in strength. The lower end of the rods column 10, rigidly connected to the traverse, is connected to the pump piston. The front and rear drive racks, pivotally connected to the frame brackets, are interconnected by front traction bars with right and left threads each.

In addition, the rear pillars with the help of the rear traction rods are also pivotally connected to the frame brackets. Front and rear traction rods provide a change in the angles of inclination of the front and rear racks. These racks can be made telescopic to adjust the height of the stand depending on the stroke of the rod suspension. The lower ends (base) of the front and rear pillars are attached to the bracket on the hinged support with the possibility of deflecting to the right or left with the front rods to free space around the wellhead during repair and to precisely control the trajectory of the rods suspension point along the vertical line. In addition, the connection of the pillars to the bracket on the hinged support makes it possible to completely unfold the front and rear racks of mechanical drive when transported to the installation site. To reduce the load on the elements of the transforming mechanism by means of a flexible link to the traverse, a movable counterweight is additionally secured.

\subsection{Kinematic study of a new design of a beamless sucker-rod pump}

It is known that the laws of motion of the rod suspension point depend on the kinematic scheme of the used mechanical drive for sucker-rod pumps. Therefore, the main goal of the kinematic analysis of any hinged mechanism is to determine the positions, velocities and accelerations of its various nodes and points $[8,10,11]$. If the length of the crank $r_{1}$ is known, then the displacement of the rod suspension point will be: $S=2 r_{1}$ as seen in Fig. 2 .

As can be seen from the diagram, the movement of the suspension point of the rod will be equal to the movement of the corresponding point of the rope. In the kinematic study, the length of the crank $A B=r_{1}$, the angle of its rotation $\varphi_{1}$, the radius of the block $D C=R_{b}$, the coordinates of the points $A(0,0), D(a, b)$ and the length of the rope $L$ are specified. The purpose of the kinematic study is to determine the displacements, velocities and accelerations of the suspension point of the $\operatorname{rod} \mathrm{E}$, depending on the angle of the crank (as a function of time). As can be seen from the scheme of the installation, the displacement of the point $\mathrm{E}$ uniquely depends on the distance $\mathrm{BC}$ and the arc 
length of the block $C F$, covered by the rope, i.e., $S_{E}=f(B C+C F)$.

\section{Solution of the problem}

To solve the problem, first need to determine the distance between the points $B$ and $C$, that is, from the point of contact of the rope with the crank to the point of contact of the rope with the block, and then the arc length of the block covered by the rope from point $C$ to point $F$.

Since the angle $\varphi_{1}$ is known:

$x_{1}=r_{1} \cos \varphi_{1}, y_{1}=r_{1} \sin \varphi_{1}$

The length of the straight line $B C$ tangent to the radius $R_{b}$ from the given point $B\left(x_{1}, y_{1}\right)$ will be [9]:

$$
B C=\sqrt{\left(x_{2}-x_{1}\right)^{2}+\left(y_{2}-y_{1}\right)^{2}}
$$

To determine the unknown coordinates $x_{2}$ and $y_{2}$ of the point $\mathrm{C}$, which is formed by touching the line $B C$ with a circle of radius $R_{b}$, we write the equation of the circle:

$$
(x-a)^{2}+(y-b)^{2}=R_{b}^{2}
$$

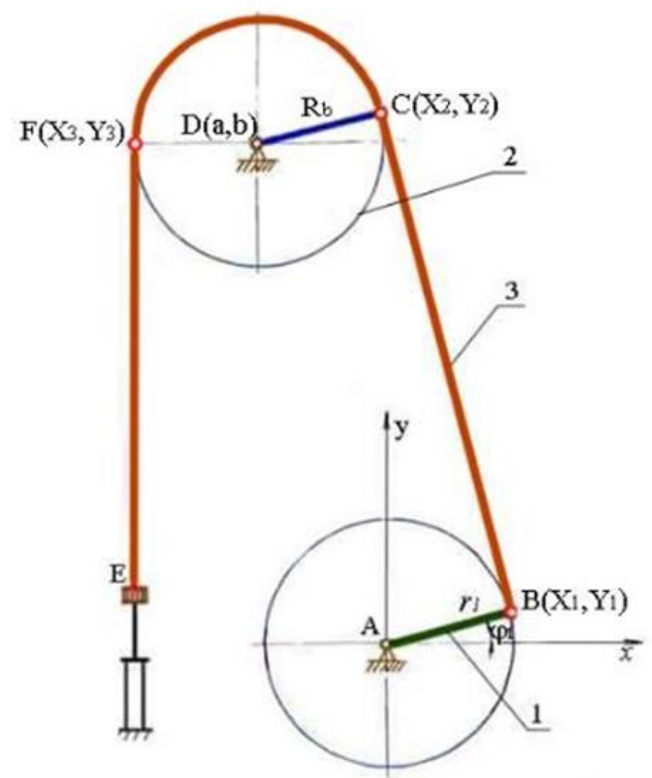

Fig. 2. Kinematic scheme of the transforming mechanism of a new constructive solution of a beamless sucker-rod pump where $\mathrm{a}$ and $\mathrm{b}$ are the coordinates of the center of the circle of radius $R_{b}$. The equation of the line $B C$ passing through the point:

$y-y_{1}=k\left(x-x_{1}\right)$

Since this line passes through the point $C\left(x_{2}, y_{2}\right)$, the correlations $\mathrm{x}_{2}$ and $\mathrm{y}_{2}$ must satisfy Eq. (4):

$y_{2}-y_{1}=k\left(x_{2}-x_{1}\right)$

where $k$ is coefficient of angle. To find this coefficient, we find the derivative of Eq. (3) in an implicit form:

$$
y^{\prime}=\frac{a-x}{y-b}
$$

From Eq. (5)

$$
k=\frac{y_{2}-y_{1}}{x_{2}-x_{1}}
$$

On the other hand

$$
k=f^{\prime}\left(x_{2}\right)=\frac{a-x_{2}}{y_{2}-b}
$$

Hence:

$$
\frac{a-x_{2}}{y_{2}-b}=\frac{y_{2}-y_{1}}{x_{2}-x_{1}}
$$

Considering

$$
\left(x_{2}-a\right)^{2}+\left(y_{2}-b\right)^{2}=R_{b}^{2}
$$

We find solving Eq. (9) from the system of equations:

$\left\{\begin{array}{l}x_{2}^{2}+y_{2}^{2}-x_{2}\left(a+x_{1}\right)-y_{2}\left(b+y_{1}\right)+a x_{1}+b y_{1}=0 \\ x_{2}^{2}+y_{2}^{2}-2 x_{2} a-2 y_{2} b+a^{2}+b^{2}-R_{b}^{2}=0\end{array}\right.$

Using the program MATLAB, we determine the coordinates $x_{2}$ and $y_{2}$, then, depending on the angle of rotation of the crank $\varphi_{1}$, we determine the required distance $\mathrm{BC}$ and the displacement of the point $\mathrm{E}$

$$
S_{E}=\left(B C_{\max }+C F\left(\varphi_{1}\right)\right)-\left(B C\left(\varphi_{1}\right)+C F\left(\varphi_{1}\right)\right)
$$


where $C F$ is the arc length of the block covered by the rope

$$
C F=\int_{x_{2}}^{x_{3}} \sqrt{1-\left(\mathrm{f}^{\prime}(\mathrm{x})\right)^{2}} d x
$$

To find $y^{\prime}(x)$, we take $f^{\prime}(x)=y^{\prime}(x)$ nd solve the equation of the circle (3) with respect to $y$

$$
y=b \pm \sqrt{R_{b}^{2}-x^{2}+2 a x-a^{2}}
$$

Differentiating this equation with respect to $x$, we obtain.

Then Eq. (13) takes the form

$$
\begin{aligned}
C F & =\int_{x_{2}}^{x_{3}} \sqrt{1+\frac{(a-x)^{2}}{R_{b}^{2}-x^{2}+2 a x-a^{2}}} d x \\
& =\int_{x_{2}}^{x_{3}} \frac{1}{\sqrt{1-\left(\frac{x-a}{R_{b}}\right)^{2}}} d x
\end{aligned}
$$

Assuming $t=\frac{x-a}{R_{b}}, d t=\frac{d x}{R_{b}}$ and $d x=R_{b} d t$, we get:

$$
\begin{aligned}
C F & =\int_{x_{2}}^{x_{3}} \frac{1}{\sqrt{1-t^{2}}} R_{b} d t=\left.R_{b} \arcsin t\right|_{x_{2}} ^{x_{3}} \\
& =\left.R_{b} \arcsin \frac{(x-a)}{R_{b}}\right|_{x_{2}} ^{x_{3}} \\
& =R_{b}\left[\arcsin \frac{\left(x_{3}-a\right)}{R_{b}}-\arcsin \frac{\left(x_{2}-a\right)}{R_{b}}\right]
\end{aligned}
$$

\section{Numerical computations}

To confirm the obtained analytical dependencies, a numerical experiment was performed with the following data: $r_{1}=0.5 \mathrm{~m}, R_{b}=1 \mathrm{~m}, b=3.5 \mathrm{~m}, a=$ $1.8 \mathrm{~m}$, and $\omega_{1}=1.256 \mathrm{rad} / \mathrm{s}$. The results are given in Table 1 and Figs. 3-5. To determine the movement, velocity and acceleration of the rod suspension point depending on the crank angle $\varphi_{1}$, the point of calculation is taken to be $\varphi_{1}=240^{\circ}$. As can be seen from the deviation graphs of actual movements, velocities and accelerations, the rod suspension points, depending on the crank angle, are close to the parameters of ideal harmonic laws.

When the rod suspension point moves up and down, the periods of displacements are subject to harmonic laws, however, the amplitude of displacements when moving upwards is approximately $8-15 \%$ greater than when moving downward.

When the crank is turned through the angle of $\varphi_{1}=90^{\circ}$, the point of suspension of the rod passes more than half of its stroke $(\mathrm{S}=506.7669 \mathrm{~mm})$ and when the crank is turned to the second quarter of revolution by $r / 2(\mathrm{~S}=456.9165 \mathrm{~mm})$. This is explained by the fact that this mechanism is a positive de-axial, that is, in the de-axial mechanism, the duration of the forward and reverse stroke is different. Therefore, when the crank is rotated clockwise (the wellhead on the left), the upward stroke is faster than the down stroke, and when the crank is rotated counterclockwise, the upstream travel takes longer than the down stroke.

From the graphic image in Fig. 4, it is seen that the maximum value of the rod suspension point velocity corresponds to the angle of $\varphi_{1}=90^{\circ}$, thus, $\mathrm{V}=0.699725 \mathrm{~m} / \mathrm{s}$. For $\varphi_{1}=0^{\circ}$ and $\varphi_{1}=180^{\circ}$, the speed of the rod suspension point is zero, because at these points the direction of travel of the suspension point of the rod changes.

As can be seen from the graphical image in Fig. 5, the acceleration of the rod suspension points is harmonic functions of the angle $\varphi_{1}$. The crank angle $\varphi_{1}$, at which the acceleration of the rod suspension point is zero, is equal to the angle at which the piston speed has the maximum value.

\section{Conclusions}

The aim of the present study is to develop a new design solution for a beamless sucker-rod pump for oil production, characterized by small dimensions and metal consumption, less energy consumption, better dynamic characteristics. The results of the analytical kinematical studies and numerical calculations of the new beamless sucker-rod pump are presented. 
Table 1. Determination of the movement, velocity and acceleration of the rod suspension point, depending on the crank angle $\varphi_{1}$

\begin{tabular}{|c|c|c|c|c|c|c|c|c|c|c|}
\hline$\varphi_{1}$ & $x_{1}$ & $y_{1}$ & $x_{2}$ & $y_{2}$ & $x_{3}$ & $B C$ & $C F$ & $S, \mathrm{~mm}$ & $V, \mathrm{~m} / \mathrm{s}$ & $a, \mathrm{~m} / \mathrm{s}^{2}$ \\
\hline 0 & 500 & 0 & 803.6 & 3584 & 2800 & 4321.025 & 3056.714 & 0 & 0 & 1.172692 \\
\hline 15 & 482.96 & 129.4 & 804.3 & 3592 & 2800 & 4299.701 & 3048.823 & 21.32368 & 0.102615 & 1.062023 \\
\hline 30 & 433.01 & 250 & 806.1 & 3610 & 2800 & 4248.363 & 3031.083 & 72.66168 & 0.247052 & 1.028730 \\
\hline 45 & 353.55 & 353.55 & 809.5 & 3638 & 2800 & 4169.419 & 3003.643 & 151.6063 & 0.409902 & 0.984300 \\
\hline 60 & 250 & 433.01 & 814.9 & 3672 & 2800 & 4066.962 & 2968.751 & 254.0628 & 0.553047 & 0.865198 \\
\hline 75 & 129.4 & 482.96 & 2496.8 & 2783 & 2800 & 3959.662 & 799.8699 & 361.3623 & 0.656353 & 0.624402 \\
\hline 90 & 0 & 500 & 2474.7 & 2762 & 2800 & 3814.258 & 830.2382 & 506.7669 & 0.699725 & 0.262145 \\
\hline 105 & -129.4 & 482.96 & 2458.5 & 2747 & 2800 & 3679.374 & 851.9724 & 641.6513 & 0.675099 & -0.148840 \\
\hline 120 & -250 & 433.01 & 2449.6 & 2740 & 2800 & 3550.867 & 863.7381 & 770.1575 & 0.618405 & -0.342670 \\
\hline 135 & -353.6 & 353.55 & 2448.2 & 2739 & 2800 & 3438.737 & 865.5781 & 882.2874 & 0.539599 & -0.576302 \\
\hline 150 & -433 & 250 & 2453.6 & 2743 & 2800 & 3352.660 & 858.465 & 968.3644 & 0.414225 & -0.757780 \\
\hline 165 & -483 & 129.4 & 2474.8 & 2762 & 2800 & 3300.513 & 830.1027 & 1020.512 & 0.250946 & -0.986890 \\
\hline 180 & -500 & 0 & 2480.4 & 2767 & 2800 & 3287.685 & 822.4880 & 1033.34 & 0.061732 & -1.143650 \\
\hline 190 & -483 & -129.4 & 2498.9 & 2785 & 2800 & 3315.451 & 796.9380 & 1005.573 & -0.133620 & -1.180740 \\
\hline 210 & -433 & -250 & 2519.1 & 2805 & 2800 & 3381.048 & 768.2900 & 939.977 & -0.315670 & -1.100330 \\
\hline 225 & -353.6 & -353.6 & 2539.7 & 2827 & 2800 & 3477.877 & 738.1719 & 843.1478 & -0.465970 & -0.908440 \\
\hline 240 & -250 & -433 & 2559.7 & 2850 & 2800 & 3597.235 & 707.9447 & 723.7903 & -0.574380 & -0.655270 \\
\hline 255 & -129.4 & -483 & 824.8 & 3721 & 2800 & 3729.219 & 2918.419 & 591.8062 & -0.635140 & -0.367260 \\
\hline 270 & 0 & -500 & 818.5 & 3692 & 2800 & 3864.108 & 2948.941 & 456.9165 & -0.649120 & -0.084520 \\
\hline 285 & 129.4 & -483 & 813.3 & 3663 & 2800 & 3992.725 & 2978.316 & 328.2994 & -0.618940 & 0.1824520 \\
\hline 300 & 250 & -433 & 809.3 & 3636 & 2800 & 4107.467 & 3005.105 & 213.5577 & -0.552170 & 0.4035780 \\
\hline 315 & 353.55 & -353.6 & 806.5 & 3613 & 2800 & 4201.791 & 3027.513 & 119.2341 & -0.453910 & 0.593890 \\
\hline 330 & 433.01 & -250 & 804.6 & 3596 & 2800 & 4270.865 & 3045.639 & 50.15945 & -0.332410 & 0.734395 \\
\hline 345 & 482.96 & -129.4 & 803.7 & 3586 & 2800 & 4311.183 & 3055.543 & 9.841723 & -0.194020 & 0.836432 \\
\hline 360 & 500 & 0 & 803.6 & 3584 & 2800 & 4321.025 & 3056.714 & 0 & 0 & 1.172692 \\
\hline
\end{tabular}

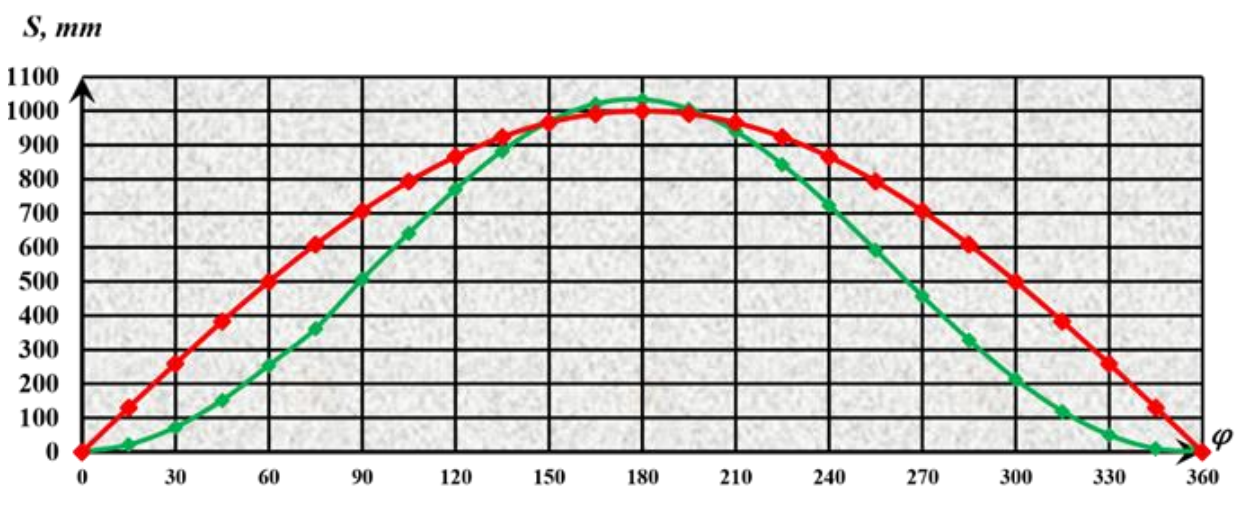

$\multimap$ real curve $\rightarrow$ ideal curve

Fig. 3. The change of movement of the rod suspension point depending on the crank angle 


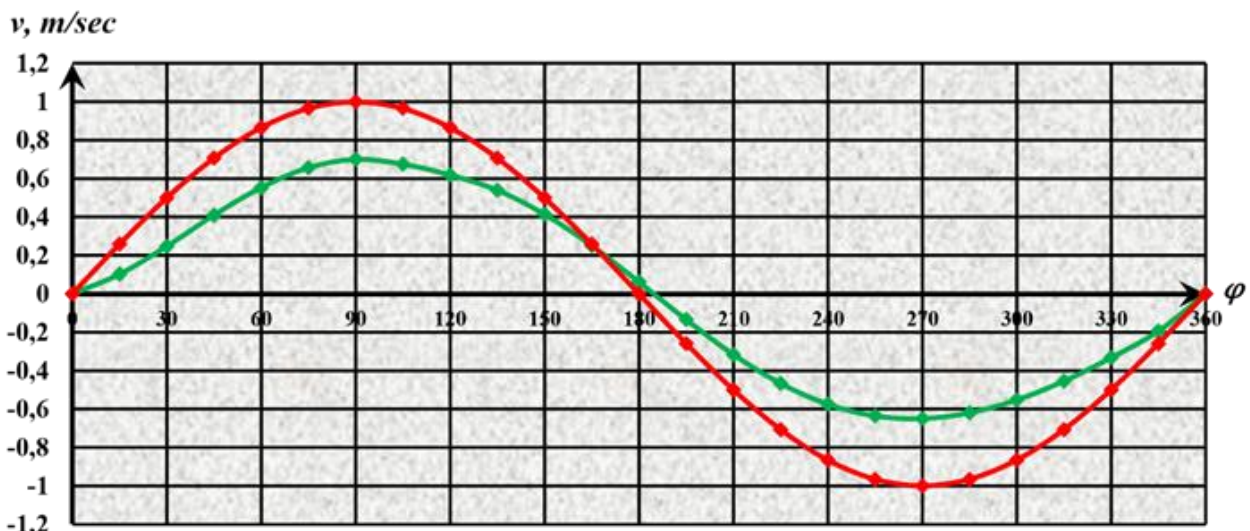

$\multimap$ real curve $\quad \rightarrow$ ideal curve

Fig. 4. The change of speed of the rod suspension point depending on the crank angle

$$
a, m / s^{2}
$$

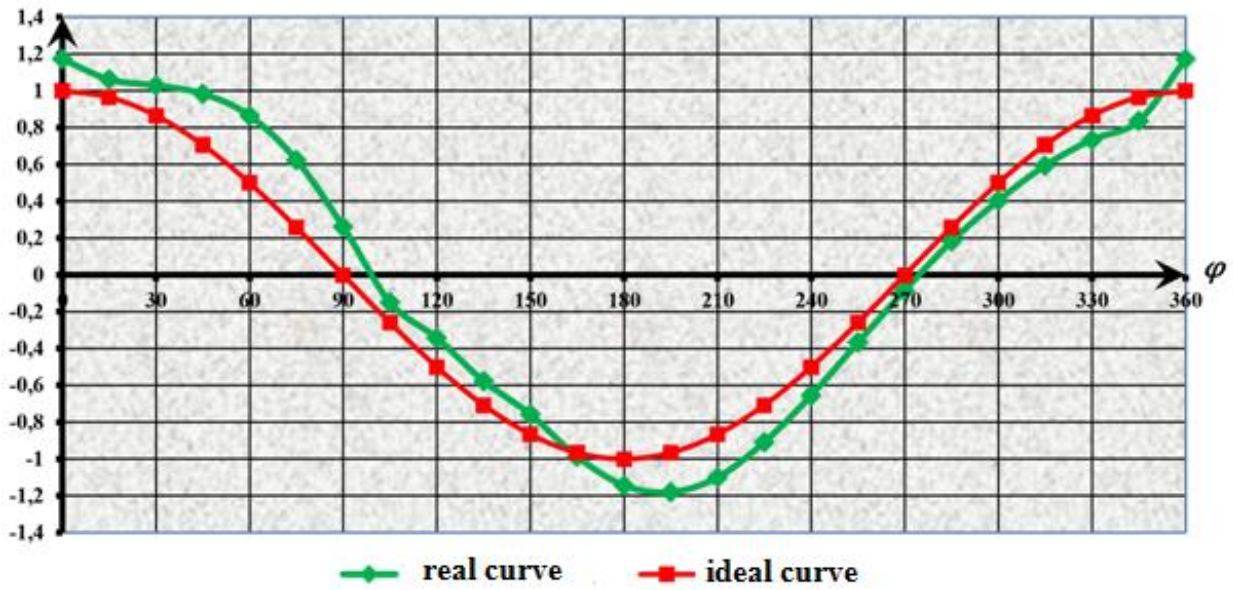

Fig. 5. The change of acceleration of the rod suspension point depending on the crank angle

The results support the following conclusions:

a) Analytical dependencies are obtained to determine the basic kinematic characteristics of the de-axial transforming mechanism of a new design of a beamless sucker-rod pump.

b) Using the proposed mechanism with a positive de-axial when the crank is rotated counterclockwise helps reduce the inertial component of the load on the suspension point of the rods during the up stroke.

c) In the de-axial mechanism, the duration of the forward and reverse stroke is different: by changing the duration of the idling or working stroke, it is possible to regulate the performance or provide more favorable operating conditions for the drive.

\section{References}

[1] Laine RE, Cole DG, Jennings JW. Production Technology: Harmonic Polished Rod Motion. Paper SPE 19724 presented at the 64th Annual Technical Conference and Exhibition of the SPE, 8-11 Oct 1989, San Antonio, Texas.

[2] Byrd, JP. History, background, and rationale of the mark II, beam type, oil field pumping unit. Proc. 37th Annual Southwestern Petroleum Short Course, 272-91, 1990.

[3] Beck Th, Peterson RA. Comparison of the performance of linear actuator versus walking beam pumping systems. Proc. 56th Annual Southwestern Petroleum Short Course, 143-165, 2009. 
[4] Takacs G (2014) Exact kinematic and torsional analysis of rotaflex pumping units. Journal of Petroleum Science and Engineering.;115: 11-16.

[5] Abdullayev AI, Najafov AM (2006) Analytical kinematics of the geared linkwork of the new constructive decision of a mechanical drive for sucker-rod pumps. Azerbaijan oil industry, Baku/Azerbaijan 4: 52-56 (in Russian).

[6] Abdullayev AI, Albers A, Najafov AM (2006) Estimation of inertial load in new constructive decision of mechanical drive for sucker-rod pumps. Azerbaijan oil industry, Baku/Azerbaijan 9: 46-49 (in Russian).
[7] Abdullayev AI, Najafov AM. Three-stage double-flow cylindrical gear/Patent № 017053 B1, F16H 1/20 ЕАПО, Moscow/Russia, Bulletin, 9: 1-4, 2012 (in Russian).

[8] Artobolevsky II. Theory of Machines and Mechanisms. Moscow, Nauka, 1988.

[9] Vygodsky MYa. Handbook of Mathematics. Moscow: AST: Astrel, 2010.

[10] Aliverdizade KS. Technology and technique of oil and gas recovery. Moscow/Russia, 1971.

[11] Najafov AM. The exploratory designing of mechanical drive of succer-rod pumps. Saarbrücken/Germany, Palmarium Academic Publishing, 2013. 